\title{
Flexibility in work time impacts workers' lunch habits
}

\section{Abstract}

Background: Workplaces have been highlighted as a promising place to promote healthy food choices because of the recurrence of eating occasions (Wanjek, 2005). Beyond interventional research promoting healthier food choices in workplace restaurants, much remains to be understood regarding the specificities of eating choices of individuals during their worktime. Specifically, although working conditions seem likely to play a key role in workers' eating choices (Raulio et al., 2007), the nature and extent of this association are unclear. Worktime organisation and workers' control over their own schedule have been proposed to account for differences in the way workers interact with their workplace food environment (Massey \& Saulais, in press; Raulio et al., 2012).

Objectives: The objective of this research is to investigate the relationships between two time-related working conditions, schedule flexibility and control over one's schedule, and workers' lunch habits.

Methodology: An ad-hoc online questionnaire was developed partly after the French Working Conditions survey. 1,139 French wage-earning workers, recruited from an online survey panel, were asked about their time-related working conditions, their behavior during lunch breaks over a two-week period (including non-food related behaviors such as taking care of personal matters during break) and their subjective experience of these lunch breaks in relation with time (such as feeling rushed during lunch break).

Results: Workers with more flexibility in their schedule were more likely to report using lunch breaks as an adjusting variable in their workday, either by reducing it to compensate for their workload $(\mathrm{p}=.0001)$ or to attend to personal matters $(\mathrm{p}=.04)$. Perceived control over worktime organization also affected eating strategies during lunchtime.

Conclusion: This research is one of the first to investigate possible relationships between working conditions and workers' lunch habits. Although results provide support to a link between time-related working conditions and lunch behaviors, socioeconomic status (SES), which have been shown to impact workers' behaviors, could be a confounding factor (Massey \& Saulais, in press), whose effect needs to be further investigated in controlled conditions.

\section{Conflict of Interest}

research partly funded by Elior entreprise and Apicil 